\title{
Health Text Messaging Intervention to Increase Fruit and Vegetable Intake ${ }^{\dagger}$
}

\author{
Alicia Clark and Maria Anna Choukri * \\ Department of Nursing, Midwifery, and Allied Health, Ara Institute of Canterbury, Christchurch 8140, \\ New Zealand; alielizabethclark@gmail.com \\ * Correspondence: maria.choukri@ara.ac.nz \\ + Presented at the 2018 Nutrition Society of New Zealand Annual Conference, Auckland, New Zealand, \\ 28-30 November 2018.
}

Published: 5 March 2019

Background: Fruit and vegetables are an important part of a healthy diet. However, only $40 \%$ of New Zealand adults currently meet the Ministry of Health recommended five or more servings each day. Individuals aged 19-30 years are the least likely to meet this recommendation. Evidence shows that text message based mHealth interventions can improve multiple health behaviours, such as smoking cessation and diabetes control. This study investigated the effect of a text message based mHealth intervention on increasing self-reported fruit and vegetable intake.

Methods: Ara Institute of Canterbury students from a level three communications course $(n=$ 26) were randomly assigned to either a control or intervention group in a two week randomised controlled trial study. Participants reported their fruit and vegetable intake both pre and post intervention through a dietary recall style questionnaire focusing on fruit and vegetable intake over the previous two weeks. Participants in the control group received a text message at 10 am daily detailing the positive health effects of meeting the Ministry of Health recommended fruit and vegetable intake each day. Participants in the control group received no text messages.

Results: At baseline, participants reported consuming 3.0 serves of fruit and vegetable per day in the intervention group and 3.9 serves a day in the control group on average. Following the intervention, participants in the intervention group reported a significantly higher average fruit and intake at 4.2 servings a day $(p<0.05)$. There was no significant increase in the control group's reports of fruit and vegetable intake.

Conclusions: This study showed significant improvement in students' self-reported fruit and vegetable intake when exposed to a mHealth based text message intervention. Therefore, the use of a text message based mHealth intervention may be an effective strategy to motivate young adults to increase their fruit and vegetable intake in New Zealand.

(C) 2019 by the authors. Licensee MDPI, Basel, Switzerland. This article is an open access article distributed under the terms and conditions of the Creative Commons Attribution (CC BY) license (http://creativecommons.org/licenses/by/4.0/). 